\title{
Large Amplitude, Short Wave Peristalsis and Its Implications for Transport
}

\author{
${ }_{3}$ Lindsay D. Waldrop ${ }^{1}$ and Laura A. Miller ${ }^{1,2}$
}

${ }_{4}^{1}$ Dept. of Mathematics, CB\#3250, Univ. of North Carolina, Chapel Hill, NC 27599

${ }_{5}^{2}$ Dept. of Biology, CB\#3280, Univ. of North Carolina, Chapel Hill, NC 27599

6 ABSTRACT

Valveless, tubular pumps are widespread in the animal kingdom, but the mechanism by which these pumps generate fluid flow are often in dispute. Where the pumping mechanism of many organs was once described as peristalsis, other mechanisms, such as dynamic suction pumping, have been suggested as possible alternative mechanisms. Peristalsis is often evaluated using criteria established in a technical definition for mechanical pumps, but this definition is based on a small-amplitude, long-wave approximation which biological pumps often violate. In this study, we use a direct numerical simulation of large-amplitude, short-wave peristalsis to investigate the relationships between fluid flow, compression frequency, compression wave speed, and tube occlusion. We also explore how the flows produced differ from the criteria outlined in the technical definition of peristalsis. We find that many of the technical criteria are violated by our model: fluid flow speeds produced by peristalsis are greater than the speeds of the compression wave; fluid flow is pulsatile; and flow speed have a non-linear relationship with compression frequency when compression wave speed is held constant. We suggest that the technical definition is inappropriate for evaluating peristalsis as a pumping mechanism for biological pumps because they too frequently violate the assumptions inherent in these criteria. Instead, we recommend that a simpler, more inclusive definition be used for assessing peristalsis as a pumping mechanism based on the presence of non-stationary compression sites that propagate uni-directionally along a tube without the need for a structurally fixed flow direction.

Keywords: Peristalsis,embryonic hearts, fluid dynamics

\section{INTRODUCTION}

Tubular pumps are found across the animal kingdom in all stages of development (e.g. Griffiths et al, 1987; Gashev, 2002; Xavier-Neto et al, 2007; Lee and Socha, 2009; Glenn et al, 2010; Xavier-Neto et al, 2010; Krenn, 2010; Greenlee et al, 2013; Harrison et al, 2013a). In all vertebrate embryos, the heart first forms as a valveless tubular pump (e.g. Taber, 2001). Similarly, the hearts of many non-vertebrate chordates such as tunicates are also valveless, tubular pumps (Anderson, 1968; Kalk, 1970; Glenn et al, 2010; Waldrop and Miller, 2015). In the last 10 years, the mechanism of pumping in tubular hearts and other tubular pumps has been described as either peristalsis or dynamic suction pumping (Forouhar et al, 2006; Taber et al, 2007; Männer et al, 2010; Maes et al, 2011).

For the case of peristalsis, a wave of active contraction propagates down the length of the tube. Since the advent of mechanical peristaltic pumps (i.e., roller pumps), the technical definition of a peristaltic pump has been refined to include these characteristics (summarized in Männer et al (2010)):

1. Peristaltic pumps are positive-displacement pumps, which displace a fixed volume to create fluid flow.

2. Peristaltic pumps have non-stationary compression sites, i.e., waves of compression that unidirectionally propagate down a flexible tube.

3. Peristaltic pumps produce continuous flow.

4. There are no structurally fixed directions of flow (e.g., there are no one-way valves), and the direction of flow is determined by the direction of the compression wave. 
5. Flow velocity is equivalent to the speed of the compression wave. Peak flow velocity does not exceed the speed of the compression wave.

6. There is a linear relationship between the frequency of the compression wave and flow rate it produces.

The principles of the technical definition are based upon a body of analytical work using small-amplitude and/or long-wave approximations of peristaltic pumping where nonlinear effects, such as inertia and large flows in the radial direction, are small (Jaffrin and Shapiro, 1971; Hanin, 1968; Shapiro et al, 1969; Fung and Yih, 1968). These results may not apply to cases when inertia is non-negligible or where the compression amplitude is not small relative to the diameter or the wavelength. These nonlinear cases do, however, characterize many biological pumps (Santhanakrishnan and Miller, 2011).

There are, however, some numerical and analytic results available for large amplitude and/or short wavelength peristalsis. Childress (2009) showed analytically for large occlusion ratios and long wave lengths that the peak flow velocity can exceed the peristaltic wave speed. Pozrikidis (1987) modeled peristalsis for relatively small wavelengths in a Stokes fluid. He found that peak flow velocities are nearly twice the wave speed for an $80 \%$ occlusion of the tube. Large amplitude, short wave peristalsis has also been studied numerically for Stokesian viscous and viscoelastic fluids. Teran et al (2008) simulated up to $50 \%$ occlusion and reported only mean flow rates for viscous and viscoelastic fluids. Aranda et al (2011) simulated high amplitude peristaltic pumping in a three-dimensional tube with a phase-shifted asymmetry. The mean flow rate approached the wave speed for full occlusion, implying that the peak speeds are higher than the wave speed given the large spatial and temporal variations in the flow. Ceniceros and Fisher (2012) also simulated viscoelastic fluids at high occlusion ratios but also only reported mean flow rates. For Newtonian fluids in nearly occluded tubes, mean flow rates approached the wave speed. Given the large spatial variations in flow as evidenced by the given vorticity plots, it is likely that peak flow speeds were much higher than the wave speed.

In contrast, dynamic suction pumping is defined by an isolated region of active contraction that is asymmetrically located in a section of flexible tube connected to relatively stiffer inflow and outflow tracts (Liebau, 1954, 1957, 1955). Passive elastic traveling waves emanate from the active contraction site, and these waves drive flow. Analytical models (Auerbach et al, 2004; Bringley et al, 2008), physical experiments (Hickerson et al, 2005a; Bringley et al, 2008), and numerical simulations (Jung and Peskin, 2000; Jung et al, 2008; Baird et al, 2014) support that this pumping mechanism can effectively transport fluid under certain conditions. Furthermore, these pumps are characterized by a nonlinear frequency-flow relationship, reversals in flow directions, and flow speeds higher than the wave speed.

Based on the technical definition of peristalsis, Forouhar et al. (2006) make the case that the zebrafish embryonic heart is not a peristaltic pump by observing the kinematics of heart compression and blood flow in vivo. They found these observations violated some of the principles that underpin peristalsis, specifically: they observed bidirectional compression and reflection waves of the heart muscle and imply that activation of the muscle was limited to one site (\#2 and \#3), the flow velocity inside the heart exceeded the compression wave speed (\#5), and there was a non-linear relationship between blood flow speed and the frequency of heart compressions (\#6). As a result of these observations, Forouhar et al (2006) reject peristalsis and suggest that the fluid dynamics of the embryonic vertebrate heart share more of the properties of dynamic suction pumping than peristalsis (a localized site of active compression is placed off-center, and the resulting traveling waves are passive elastic).

Since the publication of this paper nearly 8 years ago, some researchers have speculated that other biological pumps drive blood and other fluids using dynamic suction pumping (Davidson, 2007; Vogel, 2007; Harrison et al, 2013b). Other researchers continue to describe tubular pumping in the embryonic heart as peristalsis (Christoffels and Moorman, 2009; Postma et al, 2008; Taber et al, 2007). Some of these tubular pumps, like tunicate hearts and many embryonic hearts, violate the low-amplitude, long-wave assumptions of the technical definition of peristalsis. In many of these cases, the compression wave almost completely occludes the tube, action potentials are known to propagate the length of the entire tube by activating helically wound muscle fibers (Anderson, 1968; Kalk, 1970), and the width of the tube is not significantly longer than its length. Furthermore, these pumps are often at a scale where neither inertial nor viscous effects can be neglected.

In this paper, we use direct numerical simulation of the fully coupled fluid-structure interaction problem to quantify the flows generated by large-amplitude, short-wave peristalsis. We then evaluate 
which aspects of the technical definition of peristalsis a large-amplitude, short-wave peristaltic pump can fulfill. The simulations were done using the immersed boundary method (Peskin, 2002). The peristaltic wave was prescribed as a traveling sine wave. The length of the tube was four times the diameter, and the region of prescribed motion was centered in the middle of the tube. The ends of the elastic section were allowed to bend as determined by the coupling between the fluid and the elastic tube. We quantify peak and average fluid speeds downstream of the compression region, the pressure at the inflow and outflow points of the compression tube, and flow speeds at a point within the compression region.

\section{METHODS}

\subsection{The Immersed Boundary Method}

The modeling and numerical simulations of peristalsis were conducted with the immersed boundary method (Peskin, 2002) using the library IBAMR (Griffith, 2014). The IB method can be used either to simulate a boundary moving with a prescribed motion or for solving for the motion based on the interaction of the fluid with an elastic boundary. The following outline describes the two-dimensional formulation of the immersed boundary method, but the three-dimensional extension is straightforward. For a full review of the method, see Peskin (2002). The equations of fluid motion are given by the Navier-Stokes equations:

$$
\begin{aligned}
& \begin{array}{l}
\rho\left(\mathbf{u}_{t}(\mathbf{x}, t)+\mathbf{u}(\mathbf{x}, t)\right. \\
\cdot \nabla \mathbf{u}(\mathbf{x}, t))=-\nabla p(\mathbf{x}, t) \\
+\mu \nabla^{2} \mathbf{u}(\mathbf{x}, t)+\mathbf{F}(\mathbf{x}, t)
\end{array} \\
& \nabla \cdot \mathbf{u}(\mathbf{x}, t)=0
\end{aligned}
$$

where $\mathbf{u}(\mathbf{x}, t)$ is the fluid velocity, $p(\mathbf{x}, t)$ is the pressure, $\mathbf{F}(\mathbf{x}, t)$ is the force per unit area applied to the fluid by the immersed boundary, $\rho$ is the density of the fluid, and $\mu$ is the dynamic viscosity of the fluid. The independent variables are the time $t$ and the position $\mathbf{x}$.

The interaction equations between the fluid and the boundary are given by:

$$
\begin{aligned}
\mathbf{F}(\mathbf{x}, t) & =\int \mathbf{f}(r, t) \boldsymbol{\delta}(\mathbf{x}-\mathbf{X}(r, t)) d r \\
\mathbf{X}_{t}(r, t)=\mathbf{U}(\mathbf{X}(r, t)) & =\int \mathbf{u}(\mathbf{x}, t) \boldsymbol{\delta}(\mathbf{x}-\mathbf{X}(r, t)) d \mathbf{x}
\end{aligned}
$$

where $\mathbf{f}(r, t)$ is the force per unit length applied by the boundary to the fluid as a function of Lagrangian position and time, $\delta(\mathbf{x})$ is a two-dimensional delta function, $\mathbf{X}(r, t)$ gives the Cartesian coordinates at time $t$ of the material point labeled by the Lagrangian parameter $r$. Equation 3 applies force from the boundary to the fluid grid, and Equation 4 evaluates the local fluid velocity at the boundary. The boundary is then moved at the local fluid velocity, and this enforces the no-slip boundary condition. Each of these equations involves a two-dimensional Dirac delta function, $\delta$, which acts as the kernel of an integral transformation. These equations convert Lagrangian variables to Eulerian variables and vice versa.

The force equations are specific to the application. In a simple case where a preferred motion is enforced, boundary points are tethered to target points via springs. The equation describing the force applied to the fluid by the boundary in Lagrangian coordinates is given by:

$$
\mathbf{f}(r, t)=k_{t a r g}(\mathbf{Y}(r, t)-\mathbf{X}(r, t))
$$

where $\mathbf{f}(r, t)$ is the force per unit length, $k_{\text {targ }}$ is a stiffness coefficient of the tethering springs, and $\mathbf{Y}(r, t)$ is the prescribed position of the target boundary.

\subsection{Dimensionless Numbers}

To compare the flows of different pumps over a range of length scales and velocities, it is a useful exercise to non-dimensionalize the terms in the Navier-Stokes equations as follows,

$$
\begin{aligned}
\mathbf{x}^{\prime} & =\frac{\mathbf{x}}{L}, \\
\mathbf{u}^{\prime} & =\frac{\mathbf{u}}{U},
\end{aligned}
$$



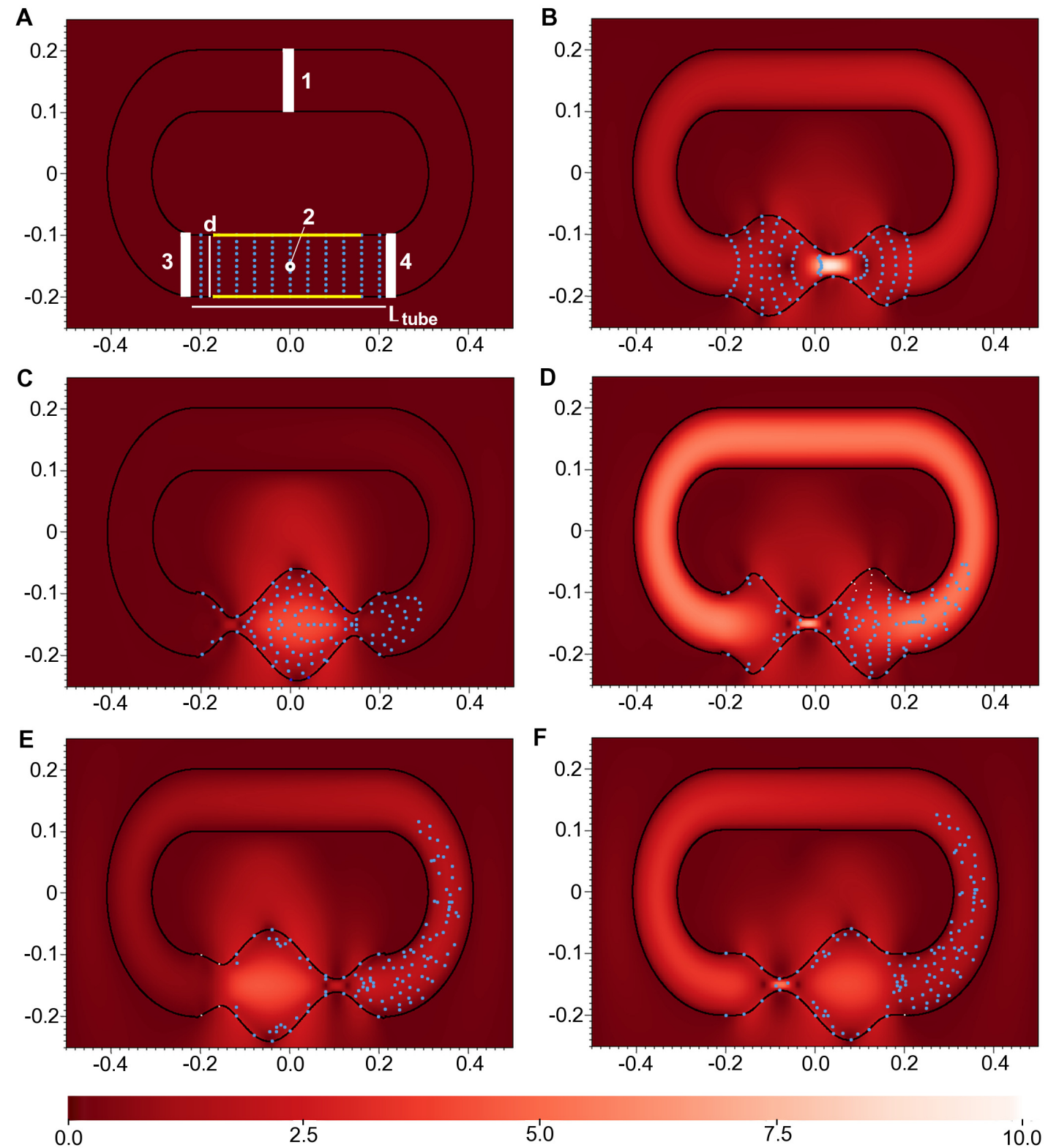

Figure 1. Panels showing racetrack circulatory system and compression region with speed across the simulation area (background color) and light-blue flow marker particles. A: Initial simulation conditions $(t=0.0)$ showing racetrack circulatory system (black lines), tube diameter $d$ (white vertical line), compression region, $L_{\text {tube }}$ (white horizontal line between white boxes 3 and 4 ), tethered region (yellow bars) and marker particles (light blue dots). Areas over which data were collected are labeled as white boxes and a white/black point (see text for details on calculations). B: Simulation at $t=0.4$. C:

Simulation at $t=0.8$. D: Simulation at $t=1.2$. E: Simulation at $t=1$.6. F: Simulation at $t=2.0$. Color scale units non-dimensional speed. 


$$
t^{\prime}=f * t,
$$

where $L, U$, and $1 / f *$ are characteristic flow length, velocity, and time scales respectively. In this paper, we choose $L$ to be the diameter of the elastic section of the tube, $f *$ to be the beat frequency of our base case, and the characteristic velocity as $U=L f *$. Note the $U$ describes the velocity in terms of heart tube lengths per beat.

The dimensionless bending stiffness of the boundary may then be calculated as

$$
k_{\text {bend }}^{\prime}=\frac{k_{\text {bend }}}{\rho U^{2} L^{3}},
$$

where $k_{\text {bend }}$ is the flexural stiffness of the boundary. The dimensionless spring stiffness may be written as

$$
k^{\prime}=\frac{k}{\rho U^{2} L},
$$

where $k$ is the spring stiffness which describes the resistance to stretching.

The dimensionless pressure can then be defined as

$$
p^{\prime}=\frac{p}{\rho U^{2}},
$$

where $p$ is the dimensional pressure and $U$ is the characteristic velocity.

Of particular relevance to internal biological flows is the Womersley number $(W o)$. The $W o$ describes to what extent unsteady effects matter in pulsatile flows. It is given by the following equation,

$$
W o=d \sqrt{\frac{\omega}{v}},
$$

where $\omega$ is the frequency of the pulse, $d$ is the diameter of the tube, and $v$ is the kinematic viscosity of the fluid. Within the context of a blood vessel, when the value of Wo is high, the velocity profile is nearly flat over most of the cross-section and there is a small region near the vessel wall known as the boundary layer where viscous effects are important. Also, the flow at the center of the tube is inertial and pulsatile. At the other low extreme end of Wo, the velocity profile over the vessel cross-section is parabolic, and the flow is quasi-steady and viscous dominated. The transient effects can be ignored when Wo is sufficiently small, generally when $W o<1$, and this is common in the case of microcirculation. Unless otherwise noted, we vary the $W o$ by changing the viscosity of the fluid only.

\subsection{Numerical Method}

We used an adaptive and parallelized version of the immersed boundary method, IBAMR (Griffith, 2014). IBAMR is a $\mathrm{C}++$ framework that provides discretization and solver infrastructure for partial differential equations on block-structured locally refined Eulerian grids (Berger and Oliger, 1984; Berger and Colella, 1989) and on Lagrangian (structural) meshes. IBAMR also includes infrastructure for coupling Eulerian and Lagrangian representations.

The Eulerian grid on which the Navier-Stokes equations were solved was locally refined near the immersed boundaries and regions of vorticity with a threshold of $|\omega|>0.1$. This Cartesian grid was organized as a hierarchy of four nested grid levels, and the finest grid was assigned a spatial step size of $d x=D / 512$, where $D$ is the length of the domain. The ratio of the spatial step size on each grid relative to the next coarsest grid was 1:4. The numerical parameters used for the simulations are given in Table 1.

\subsection{Model of Peristalsis}

A numerical model of an elastic heart tube connected to a rigid racetrack was constructed to study high amplitude peristaltic flows. The racetrack design was used for easy comparison to previous models of tubular heart pumping (e.g. Jung and Peskin, 2000; Hickerson et al, 2005b; Baird et al, 2014; Avrahami and Gharib, 2008; Lee et al, 2012). The racetrack section was constructed by connecting two sections of straight tube (one of which represents the elastic heart) to curved sections. The resting diameter of the racetrack was constant throughout its length. Dimensions and elastic properties of the racetrack are given in Table 2, and the model set up is shown in Fig. 1A. 

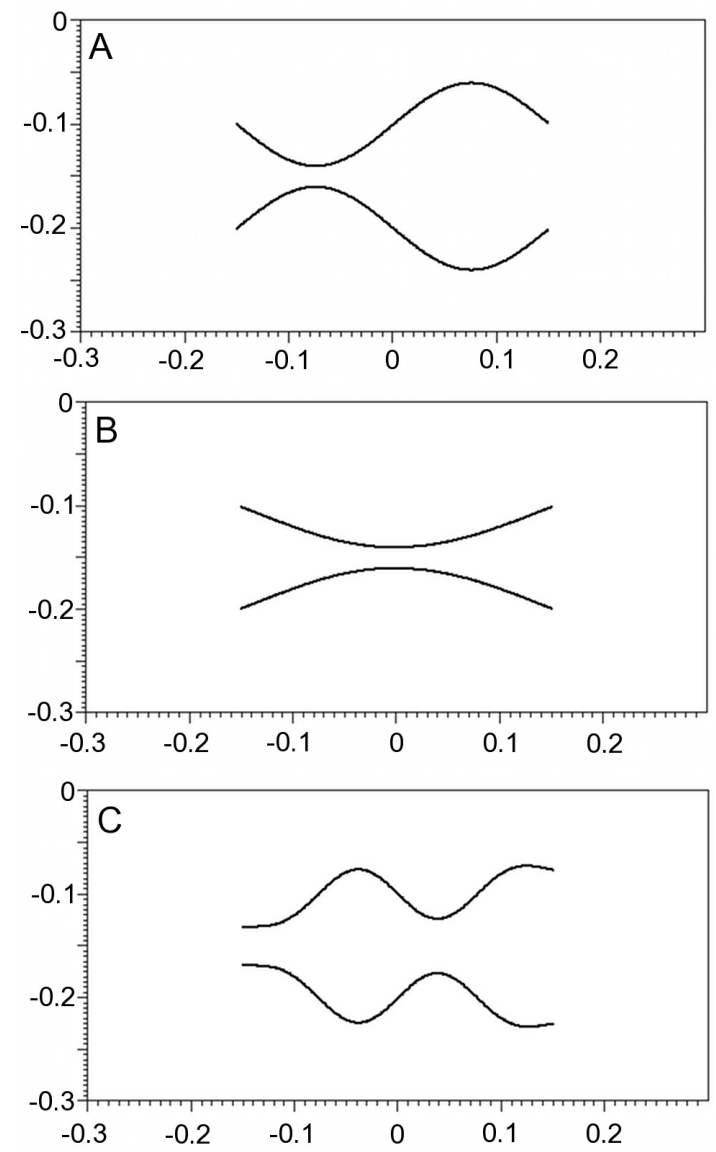

Figure 2. Panels showing no-racetrack design and several waveforms at simulation time $=2$ seconds. Waveforms are the result of keeping a constant speed of compression wave, $c=3.0$, while altering the frequency of compression wave, $f$. A: $f=1.0$ (default value), B: $f=0.5, \mathrm{C}: f=2.0$. Note that the domains in this figure have been reduce to show detail of the waveforms and do not reflect the domain on which the simulations were performed.

Table 1. Parameter values for two-dimensional immersed-boundary simulations.

\begin{tabular}{ll}
\hline Parameter & value \\
\hline Maximum time step $(d t)$ & 0.00001 \\
Minimum Eulerian spatial step $(d x)$ & 0.01952 \\
Lagrangian spatial step $(d s)$ & 0.00976 \\
Domain size $(D)$ & 10.0 \\
Refinement ratio $\left(R_{f}\right)$ & $4: 1$ \\
\hline
\end{tabular}


Table 2. Dimensionless parameter values for the peristalsis model.

\begin{tabular}{ll}
\hline Parameter & value \\
\hline Length pumping section $\left(L_{\text {tube }}\right)$ & 4.0 \\
Diameter $(d)$ & 1.0 \\
Contraction frequency $(f)$ & $0.25-3$ \\
Womersley number $(W o)$ & $0.1-50$ \\
Spring constant $\left(k^{\prime}\right)$ & $0.03-100$ \\
Bending stiffness $\left(k_{\text {bend }}^{\prime}\right)$ & $0.001-1$ \\
Wave speed $(c)$ & $1.67-6$ \\
$y$ coordinate of top of tube $\left(R_{t o p}\right)$ & -0.1 \\
$y$ coordinate of bottom of tube $\left(R_{b o t}\right)$ & -0.2 \\
Amplitude of contraction $(A)$ & $0.1 d-0.475 d$ \\
Compression ratio & $0.40-0.95$ \\
\hline
\end{tabular}

The bottom portion of the racetrack consisted of an elastic section of dimensionless length $L=4.0$ and dimensionless diameter $d=1.0$. The middle $3 / 4$ of the tube was tethered to target points that drove the peristaltic motion. Note that the tube was initialized at rest in a straight configuration. The amplitude of the peristaltic wave increased from zero to its maximum amplitude after one pulse. To ensure conservation of volume inside the racetrack during pumping, the ends of the elastic section were allowed to deform as a result of the coupling between the elastic boundary and the fluid. Note that the sides and top of the racetrack were also tethered to target points that did not move. The target point stiffness, $k_{\text {targ }}$ was set equal to the spring stiffness $k$. This parameter can be adjusted to minimize the distance between the actual boundary point and its target position. The position of the target points was determined by the following equation,

$$
y_{t o p, b o t}=R_{t o p, b o t} \pm A \sin \left(2 \pi f t+2 \pi c x_{t}\right)
$$

where $y_{\text {top,bot }}$ is the $y$-coordinate of the top or bottom of the tube, $R_{t o p, b o t}$ is the distance of the top or bottom of the tube from the horizontal centerline of the racetrack, $A$ is the amplitude of the contraction, $f$ is the frequency of contraction, $c$ is the wave speed, and $x_{t}$ is the horizontal distance from the beginning of the prescribed motion. The compression ratio gives the percent occlusion, and is equal to $2 A$.

In addition to the racetrack design, we constructed an open model of peristalsis which consisted of only the elastic heart tube on the same domain (Fig. 2). The heart tube was driven in an identical way to the racetrack design. This design allowed us to eliminate the possibility of Liebau pumping in our closed, racetrack model by removing the flexible regions of heart tube that connected the contracting region to the rigid racetrack.

\subsection{Parameter Sweeps}

We varied the values of four parameters in five separate sets of simulations. 1) Wo was changed by altering the dynamic viscosity $\mu$ of the fluid within the tube, which alters $v$ in Equation 9 since $v=\mu / \rho$, where $\rho$ is fluid density. Wo ranged from 0.1 to 10. 2) Tube occlusion was changed by altering the amplitude of contraction $A$ in Equation 10 by some factor (compression ratio), which ranged from $40 \%$ tube occlusion $($ compression ratio $=0.4)$ to nearly complete occlusion of the tube $($ compression ratio $=0.95)$. 3 ) The speed of compression wave 4) the frequency of compressions ( $f$ in Equation 10) was changed, ranging from 0.5 to 2.0, while allowing the speed of compression wave, $c$, to increase linearly. 5) The frequency of compressions ( $f$ in Equation 10) was changed while holding the speed of the compression wave, $c$, constant. Note that the no-racetrack design was used only for sweeps of compression wave frequency.

The default parameter values used for simulation sweeps other than the parameter of interest are: $W o=1, f *=1, c=3.0$, and compression ratio $=0.8$.

\subsection{Data Analysis}

For each simulation, several calculations were made on fluid flow velocity in VisIt 2.5.2 (Childs et al, 2012) (for the racetrack design), Matlab (for the no-racetrack design), and $R$ (Team, 2011) (both designs). The compression wave propagated along the compression tube from left to right which drove fluid flow in 
the counter-clockwise direction around the racetrack (Fig. 1A-F). As a result, all positive flow speeds indicate counter-clockwise flow (in the direction of the propagating compression wave) and negative values indicate clockwise flow (opposite to the direction of the propagating compression wave).

$U_{\text {avg }}$ : At each time point in the simulation, the non-dimensional magnitude of velocity $\left|\mathbf{u}^{\prime}\right|$ of fluid flow was spatially averaged across the cross-section of the upper tube of the racetrack indicated by a white box labeled 1 in Fig. 1A. These mean speeds were then temporally averaged over the entire simulation to find the average speed per simulation $U_{a v g}$, presented in Fig. 5A-C (black circles) and Fig. 8A (black and gray circles).

$U_{\max , t}, U_{\max }$ : The non-dimensional maximum magnitude of velocity across the same section of tube (white box labeled 1 in Fig. 1A) was also calculated for each time point of the simulation, $U_{\text {max }, t}$ Fig. 3 shows $U_{\text {max }, t}$ versus dimensionless time. These numbers were temporally averaged over the entire simulation to find the average maximum speed per simulation, $U_{\max }$, presented in Fig. 5A-C (white diamonds) and Fig. 8A (white and gray diamonds).

$U_{\text {peak }}$ : Using the non-dimensional, maximum speeds across the upper section of tube (white box labeled 1 in Fig. 1A) for each simulation time step, peak speeds (maximum of maximum speed) were found for each pulse. For each simulation, the peak speeds over each pulse were averaged to find the average peak speed for each simulation, $U_{\text {peak }}$.

$u_{m}, u_{x, t}, u_{x}$ : To characterize some of the dynamics in the compression region, the instantaneous, non-dimensional magnitude of velocity $\left(\left|\mathbf{u}^{\prime}\right|\right.$, speed) and $x$-component of fluid flow velocity, $u_{x, t}$, were sampled at each time at one point in the center of the compression tube, indicated by the white/black dot labeled 2 in Fig. 1A. For the $x$-component of velocity, the instantaneous component of velocity for each time step $u_{x, t}$ versus $t^{\prime}$ are presented in Fig. 4 . These speeds were then temporally averaged across the entire simulation to find the average speed or magnitude of velocity $\left(u_{m}\right)$ and average $x$-component of velocity $\left(u_{x}\right)$ for each simulation. These speeds are presented in Fig. 5D-F as black squares $\left(u_{m}\right)$ and triangles $\left(u_{x}\right)$ and in Fig. 8B as black and gray squares $\left(u_{m}\right)$ and black and gray triangles $\left(u_{x}\right)$.

$p_{\text {in }}, p_{\text {out }}, \Delta p$ : Non-dimensional pressure, $p^{\prime}$, at each time step in the simulation was spatially averaged across two cross-sections of the racetrack near the compression region: the inflow region (Fig. 1A, white box 3) and outflow region (Fig. 1A, white box 4). The inflow pressures were subtracted from the outflow pressures to find the pressure difference at each time step and averaged across the entire simulation to find the average inflow pressure $p_{\text {in }}$, the average outflow pressure $p_{\text {out }}$, and average pressure difference $(\Delta p)$ for each simulation. These pressures are presented in Fig. 7A-D as connected circles $\left(p_{\text {in }}\right)$, connected diamonds $\left(p_{\text {out }}\right)$ and dotted triangles $(\Delta p)$.

For the no-racetrack design, the average volume flow rate $v_{\text {avg }}$ was calculated using Matlab. The average $x$-component of velocity across the opening of the heart tube was calculated and then divided by the diameter of the opening at that time point. These values were then temporally averaged across simulation time to find $v_{\text {avg }}$.

\section{RESULTS}

Results discussed below are for the racetrack design unless otherwise noted.

\subsection{Womersley number}

Flow speeds in the racetrack. $U_{\max , t}$ is graphed against simulation time for several $W o$ in Fig. 3A. Note that the Wo was varied by changing the viscosity of the fluid rather than the pulse frequency and/or the wave speed. The dashed line shows the constant wave speed for these simulations. Fluid flow speeds exhibit pulsatile behavior for $W o$ of 5 and below. Unsteady behavior is observed for $W o=50$, likely due to inertial effects and the formation of strong vortices in the tube. In all cases, the maximum dimensionless flow speed is greater than the wave speed for part of the cycle.

$U_{a v g}, U_{\text {max }}$, and $U_{\text {peak }}$ are graphed against Womersley number (Wo) in Fig. 5A. Non-linear relationships exist between each of the dimensionless flow speed metrics and Wo, where the Wo was varied by changing the viscosity. $U_{\text {avg }}$ tend to steadily increase with increasing $W o$, while average peak flow speeds are greatest around $W o=1$ and 50. $U_{\text {peak }}$ are greater than the speed of the compression wave, $c$, for all Wo and show variability with simulation time for values of $W o>10$ (Fig. 3A). 
A: Womersley number

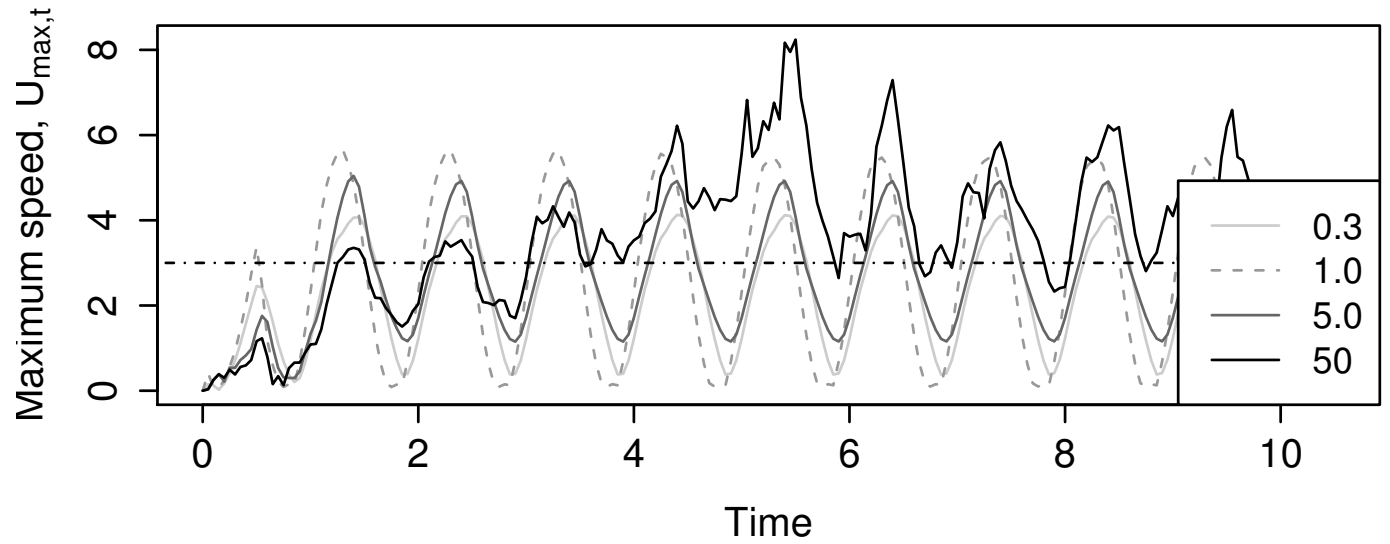

B: Compression ratio

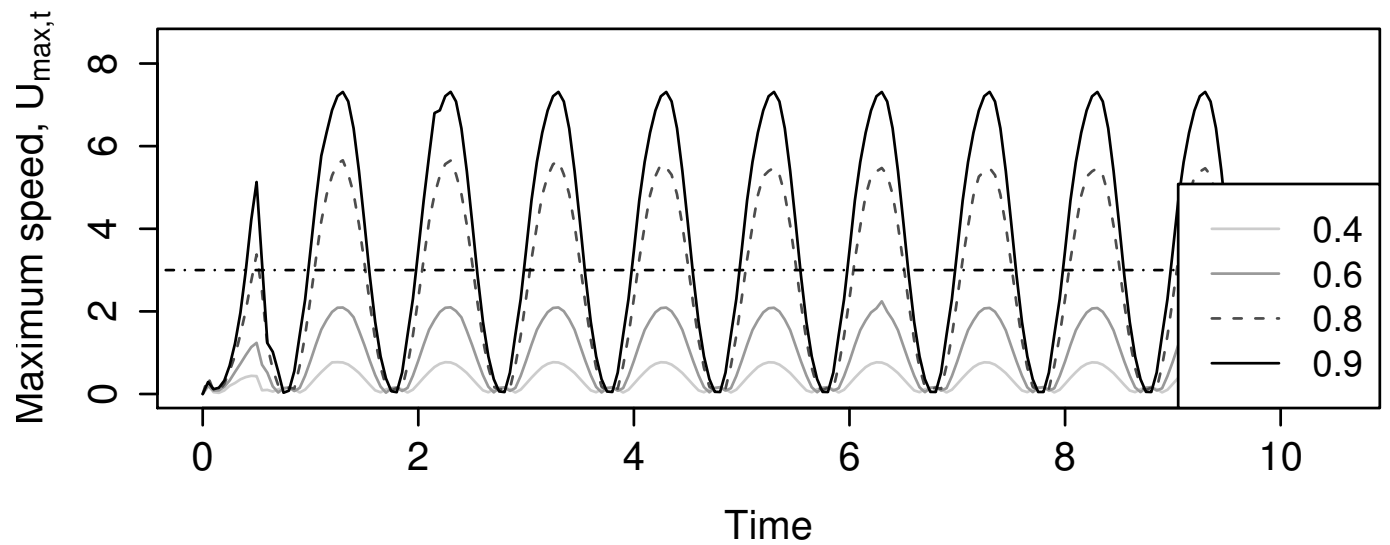

C: Wave speed

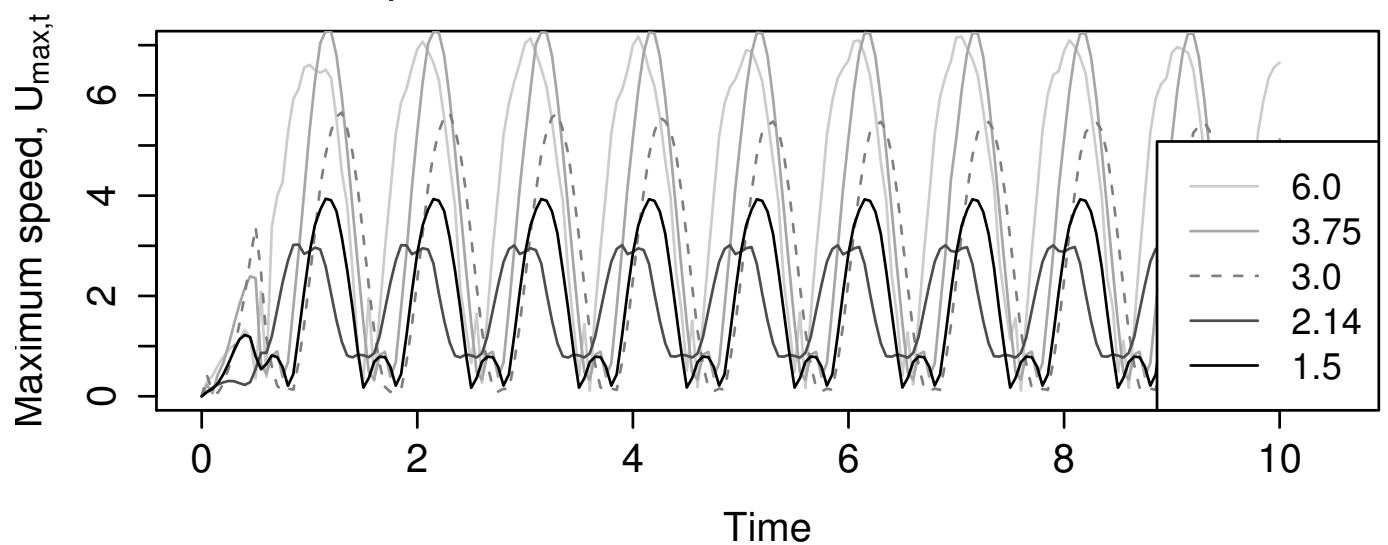

Figure 3. $U_{\max , t}$ vs. simulation time $t^{\prime}$. Dotted lines indicate default values for each parameter. Positive speed indicates movement of fluid in the counter-clockwise direction around the racetrack. Horizontal dot-dash line indicates the non-dimensional speed of the compression wave for A and B. A: Womersley number, $\mathrm{B}$ : compression ratio, $\mathrm{C}$ : non-dimensional speed of the compression wave, $c$. 

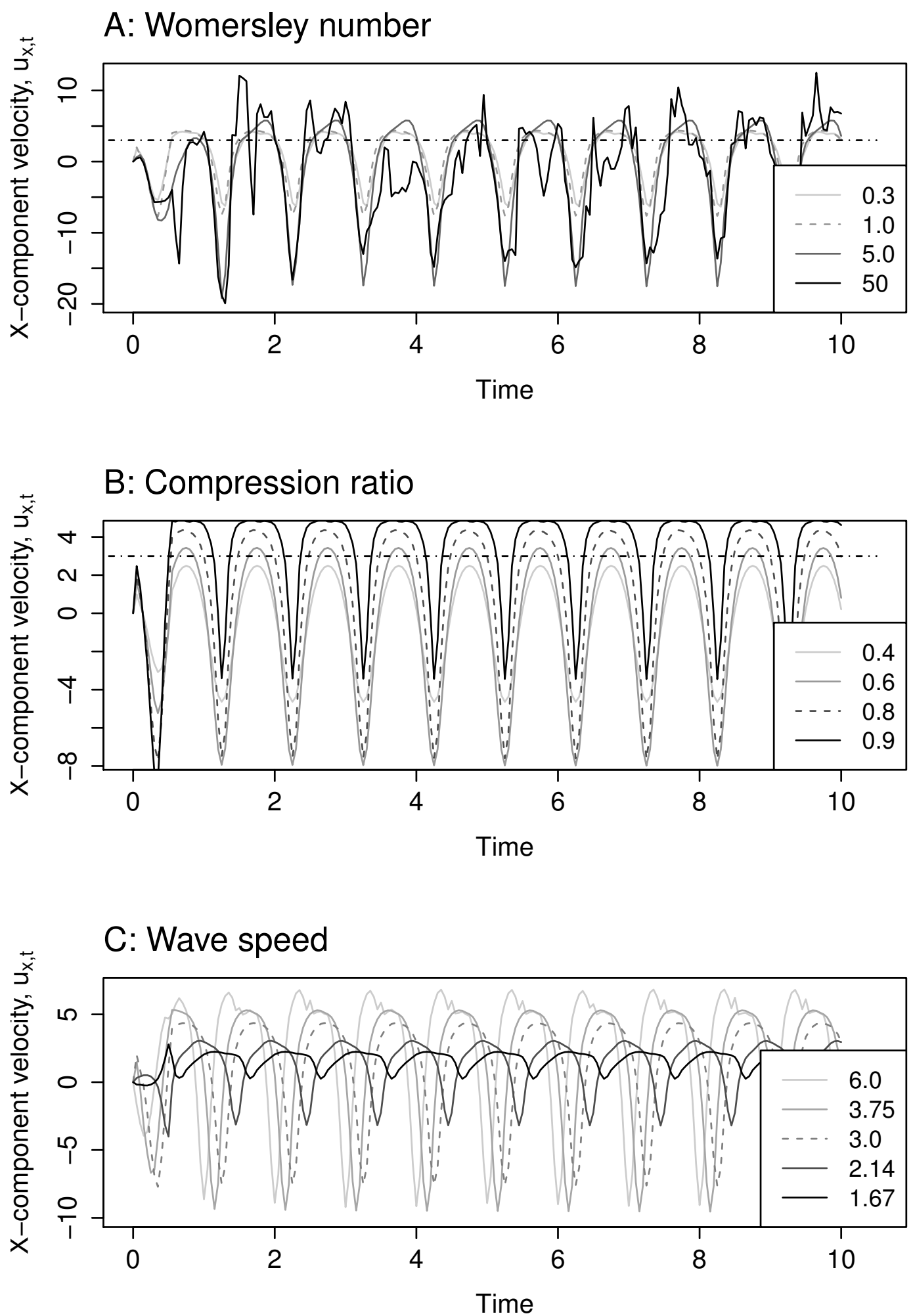

Figure 4. $u_{x, t}$ vs. dimensionless time, $t^{\prime}$, for a point at the center of the compression tube. Positive speed indicates movement of fluid in the counter-clockwise direction around the racetrack. Dotted lines indicate default values for each parameter. Horizontal dotted-dashed line indicates the non-dimensional speed of the compression wave, $c$, for A and B. A: Womersley number, B: compression ratio, C: non-dimensional compression wave speed, $c$. 

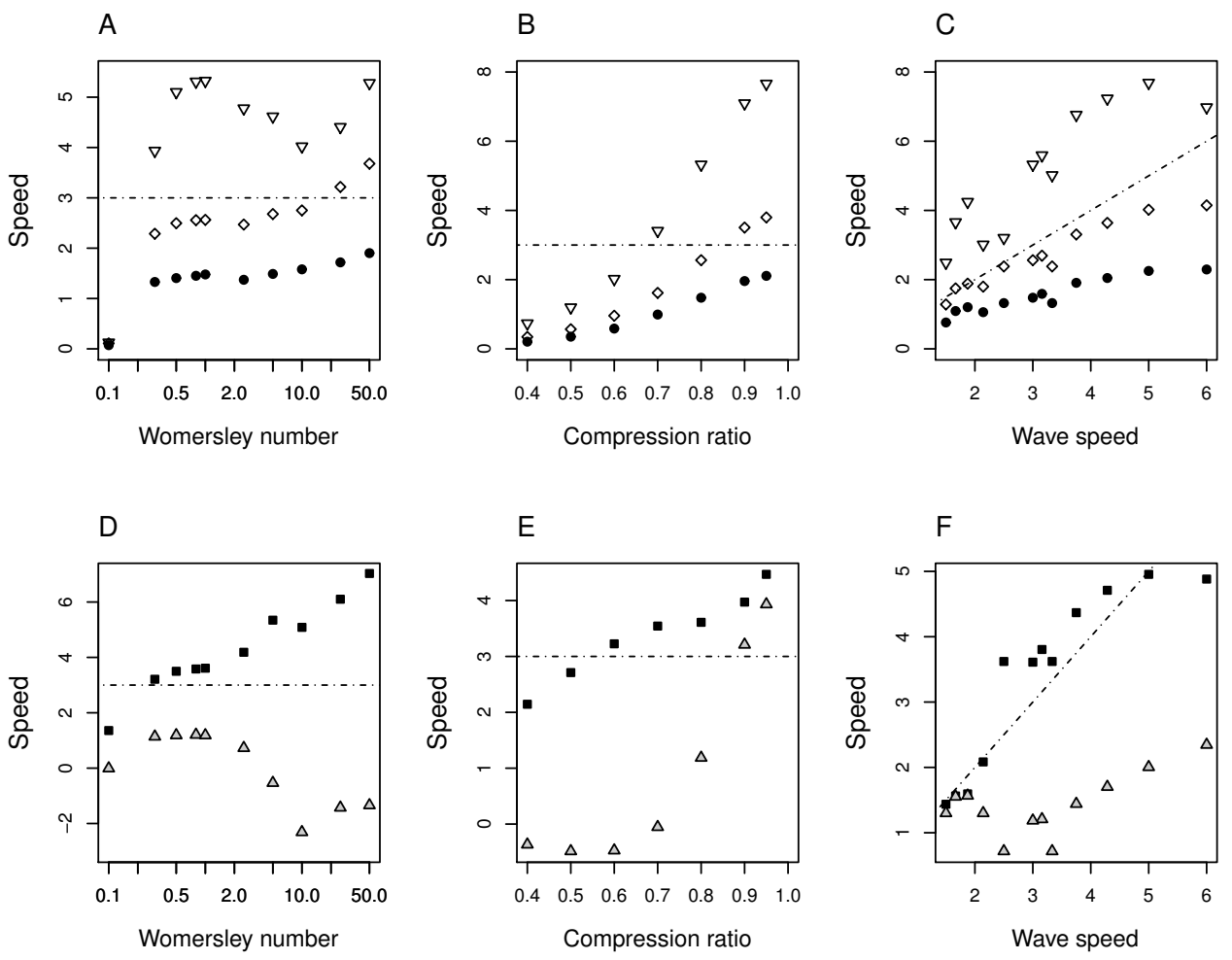

Figure 5. A-C: Non-dimensional fluid speeds within the racetrack versus parameter for $U_{\text {avg }}$ (black circles), $U_{\max }$ (white diamonds), and $U_{\text {peak }}$ (white, inverted triangles) across a section of tube (see text for details of calculations). Positive speed indicates movement of fluid in the counter-clockwise direction around the racetrack. D-F: $u_{m}$ (black squares) and $u_{x}$ (white triangles) vs. parameter for a point at the center of the compression tube. Non-dimensional compression wave speed, $c$, is noted on each plot as a dotted-dashed line. A,D: Womersley number, B,E: compression ratio, C,F: non-dimensional speed of the compression wave, $c$.

Flow speeds within the compression tube. $u_{m}$ and $u_{x}$ are plotted against $W o$ in Fig. 5D. $u_{x, t}$ is plotted against time for several simulations in Fig. 4A. The flow reversals (indicated by a change in sign) show a non-parabolic flow profile in the pumping section for $W o \geq 10$. While $u_{m}$ at this point increases with increasing $W o$, the temporally averaged value of $u_{x}$ decreases and becomes negative for $W o>1$. This indicates that flow is moving on average in clockwise direction, or opposite the direction of the propagating compression wave. This phenomenon is due to significant back flow that occurs when the tube rapidly expands behind the compression wave.

Pressure. For values $W_{o}<2$, there are large differences in pressure $(\Delta p)$. Since $W o$ is varied by changing viscosity, the $W o<1$ cases are viscous dominated, and this results in a high resistance of the fluid to move through the tube. $\Delta p$ are greatest at $W o=0.3$, with $p^{\prime}>4000$ (Fig. 7A). Of note is the case where $W o=0.1$ and the pressure difference decreases relative to $W o=0.3$. Note in Fig. 5A that the flow speed at this Wo drops to almost zero. For a compression ratio of 0.8 , the peristaltic pump is not able to drive highly viscous flow around the racetrack, and the pressure difference between the inflow and outflow tracts drops. For values $W \geq 2$, pressure differences between the two regions approach zero as the resistance to flow decreases with decreasing viscosity. Non-dimensional pressure $p^{\prime}$ is reported versus time in Fig. 6A for varying values of Wo.

\subsection{Tube Occlusion}

Flow speeds in the racetrack. $U_{\text {max }, t}$ is graphed against dimensionless time for several compression ratios in Fig. 3B. Note that $U_{\max , t}$ does not exceed $c$ for compression ratios set to 0.6 and less. For 

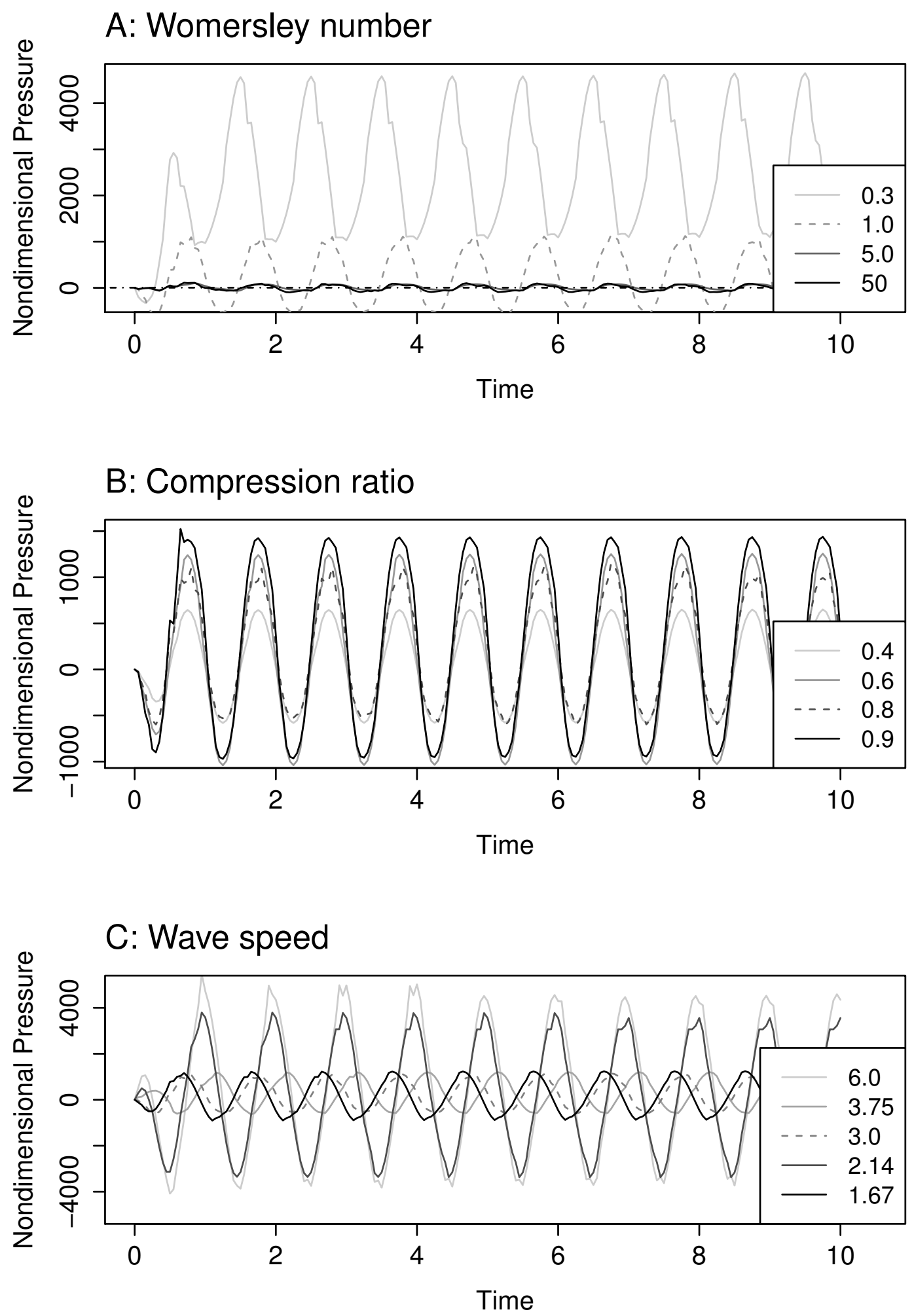

Figure 6. $p^{\prime}$ vs. dimensionless time, $t^{\prime}$, for a point at the center of the compression tube. Dotted lines indicate default values for each parameter. Horizontal dotted-dashed line indicates the non-dimensional speed of the compression wave, $c$, for A and B. A: Womersley number, B: compression ratio, C: non-dimensional compression wave speed, $c$. 
large compression ratios of $0.8-0.9, U_{\text {max. } t}$ reaches speeds of nearly double the value of $c$. In all cases considered, $U_{\max , t}$ is positive, and the flow moves in the counterclockwise direction at the top of the racetrack.

$U_{\text {avg }}, U_{\max }$, and $U_{\text {peak }}$ are graphed against compression ratio in Fig. 5B. Each metric of the flow speed has a non-linear, increasing relationship with increasing compression ratios. For compression ratios under 0.7 , corresponding to $70 \%$ tube occlusion, $U_{\text {peak }}$ does not exceed $c$ which is consistent with a low-amplitude approximation of peristalsis (Fig. 3B). However, for values 0.7 and above, the average peak flow speeds exceed $c$.

Flow speeds within the compression tube. Fig. 4B reports $u_{x, t}$ measured at the center of the compression region as a function of dimensionless time for different compression ratios. Note that in all cases, significant backflow occurs as the tube rapidly expands behind the compression wave. Backflow is minimized at the highest compression ration of 0.9 . Fig. 5E reports the temporally averaged speeds, $u_{m}$ and $u_{x}$, versus compression ratio. Higher compression ratios lead to higher forward flow speeds, as evidenced by the increase in $u_{x}$ relative to overall magnitude in Fig. 5B. $u_{x}$ exceeds the speed of the compression wave for the highest values $(\geq 0.9) . u_{m}$ starts to exceed $c$ as low as compression ratio $=0.6$ (Fig. 4B).

Pressure. Pressure differences between the inflow region (Fig. 1A at 3) and outflow region (Fig. 1A at 4) are small for lower compression ratios and grow non-linearly with increasing tube occlusion (Fig. 7B). The higher pressure differences correspond to the larger flow speeds generated by the higher compression ratios. Non-dimensional pressure $p^{\prime}$ is reported versus time in Fig. 6B for varying values of compression ratio.

\subsection{Speed of Compression Wave, $c$}

Flow speeds in the racetrack. In this set of simulations, the pulsing frequency is held constant and $c$ is varied. $U_{\max , t}$ is graphed against dimensionless time for several wave speeds in Fig. 3C. Note that changing $c$ while holding the frequency constant has the effect of changing the wave form (see Fig. 2). The base case wave speed, $c=3.0$ was halved and doubled. As expected, lower values of $c$ resulted in slow flows and higher values of $c$ resulted in faster flows.

Fig. $5 \mathrm{C}$ presents $U_{\text {avg }}, U_{\text {max }}$, and $U_{\text {peak }}$ versus $c$ for the upper section of the racetrack. All three speeds have a non-linear and increasing relationship with increasing wave speed, with average peak speeds being consistently higher than $c$. There is a small dip in fluid speeds around value $c=3.33$ which is consistent across all three measures of fluid speed.

Flow speeds within the compression tube. Fig. $4 \mathrm{C}$ reports $u_{x, t}$ as a function of dimensionless time for different wave speeds. Note that there is significant backflow for the higher wave speed cases. In Fig. 5F, the temporally averaged speeds $u_{m}$ and $u_{x}$ measured at a point within the compression tube are plotted against the wave speed. All three speed metrics have a similar non-linear relationship with $c$. Speeds increase overall with increasing $c$.

Pressure. Pressures across both the inflow region (Fig. 1A at 3) and outflow region (Fig. 1A at 4) shares a non-linear relationship with $c$, similar to the relationship seen between fluid flow speed and wave speed (Fig. 7C). Generally, increasing wave speed also increases the pressure difference between the two points. Note that the pressure difference dips around $c=3.33$, just as $U_{a v g}, U_{\max }$, and $U_{\text {peak }}$ also drop at this value of $c$. Non-dimensional pressure $p^{\prime}$ is reported versus time in Fig. 6C for varying values of $c$.

\subsection{Compression Wave Frequency}

Here we consider the following two cases: 1) frequency and wave speed are varied proportionally such that the waveform along the compression tube is unchanged, and 2) frequency is varied while the wave speed is held constant. This allows us to consider the cases when wave speed is coupled to and decoupled from changes in the pulse frequency. 
A

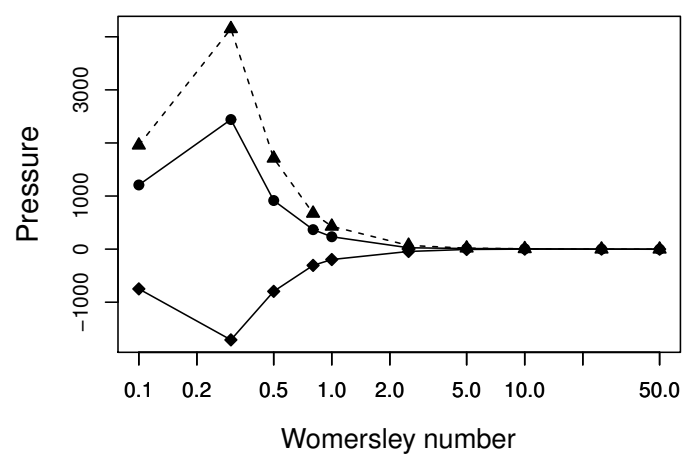

C

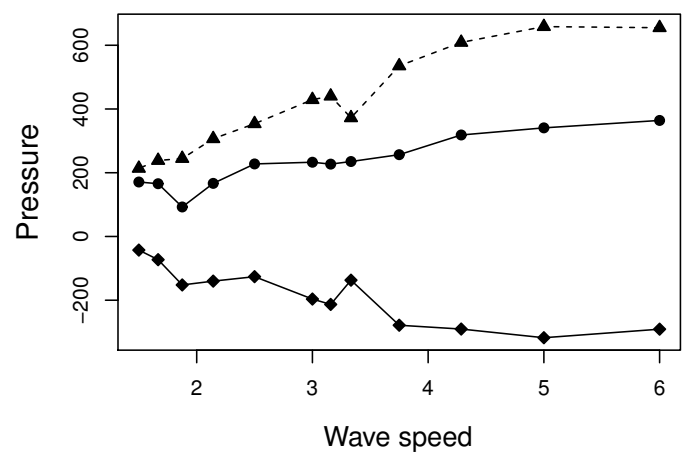

B

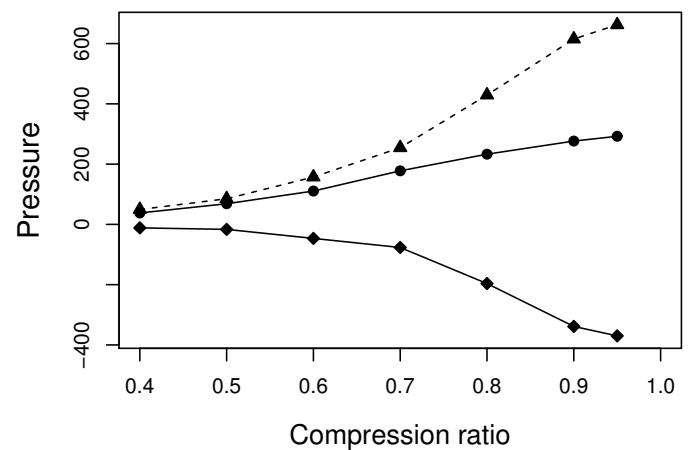

D

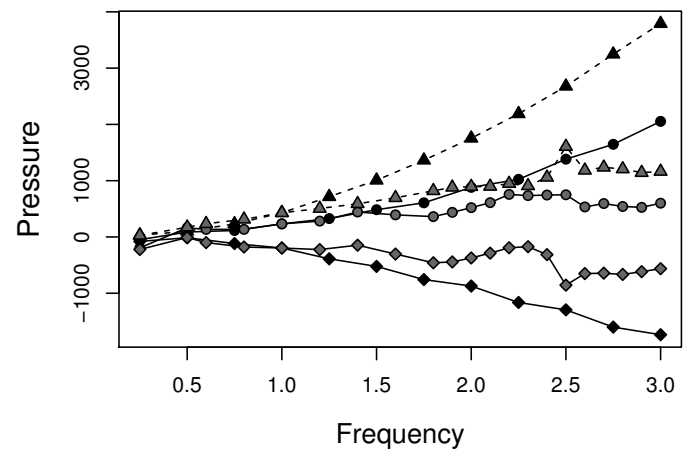

Figure 7. Pressure versus parameter across two sections of the racetrack. $p_{\text {in }}$ : circles, solid line; $p_{\text {out }}$ : black diamonds, solid line; $\Delta p$ : black triangles, dotted line. A: Womersley number, B: compression ratio, $\mathrm{C}$ : non-dimensional speed of the compression wave, $c$, and D: non-dimensional compression-wave frequency, $f$. For $\mathrm{D}$, frequencies with constant wave speed are gray and frequencies with varying wave speeds are black.

\subsubsection{Variable Wave Speed}

Flow Speed. Fig. 8A shows $U_{\text {avg }}, U_{\max }$, and $U_{\text {peak }}$ as a function of the pulse frequency, $f *$, for the racetrack design. The grey data connected by lines shows the case when the compression wave speed is allowed to change proportionally with $f *$. In this case, all measures of speed show a linear relationships with $f *$. The dashed line shows the wave speed, and $U_{\max }$ is close to the wave speed across all frequencies considered. $U_{\text {peak }}$ also varies linearly with $f *$ and is higher than the wave speed. (Fig. 8B) shows $u_{m}$ and $u_{x}$ measured in the compression region as a function of $f *$. The grey data shows the cases where $c$ varies with $f *$, and a clear linear relationship is observed. The linear relationships between flow speeds ( $U_{\text {avg }}$ and $U_{\text {peak }}$ ) and $f$ for flows generated in the no-racetrack model as well (Fig 9A). Additionally, $U_{\text {peak }}$ exceed $c$ for the no-racetrack design.

Volume Flow Rate. For the no-racetrack design, the volume flow rate, $v_{\text {avg }}$, has a linear relationship with compression frequency, $f$, when $c$ increases with increasing $f$ (Fig. 9C).

Pressure. Fig. 7D shows the relationship between pressure, $p^{\prime}$, and compression frequency, $f$, for cases when the wave speed, $c$, changes proportionally with frequency (black items). $p_{i n}^{\prime}$ shows a nonlinear but regular increase with increasing $f$, and $p_{\text {out }}$ shows a nonlinear decrease with increasing frequency. The pressure difference, $\Delta p$ between the inflow and outflow tract increases in a regular and nonlinear way with $f$. 
A

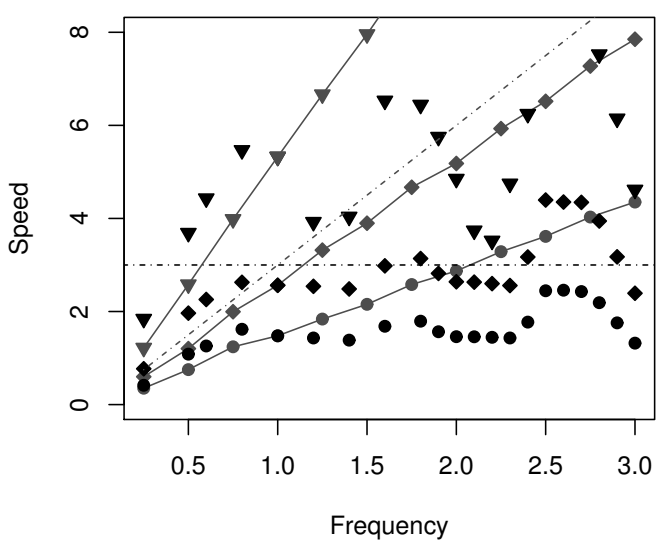

B

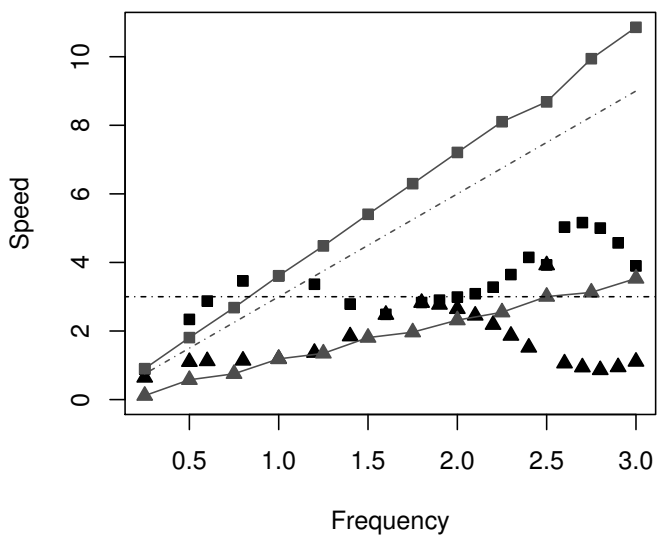

Figure 8. Non-dimensional fluid speed vs. non-dimensional compression wave frequency, $f$, for two cases: simulations with constant compression wave speed, $c$, (black items, no lines) or variable $c$ (gray, solid line). Positive speed indicates movement of fluid in the counter-clockwise direction around the racetrack. A: $U_{\text {avg }}$ (circles), $U_{\max }$ (diamonds), and $U_{\text {peak }}$ (inverted triangles) measured in the upper tube of the racetrack. (See text for description of calculations.) B: $u_{m}$ (squares) and $u_{x}$ (triangles) measured at a point within the compression region of the tube.

\subsubsection{Constant Wave Speed}

Flow speeds in the racetrack. When compression wave speed is held constant while the pumping frequency increases, all measures of fluid speed have markedly non-linear relationships with compression frequency. Note that this has the effect of changing the wave form along the length of the compression region. Within the upper section of the racetrack, $U_{a v g}, U_{\max }$, and $U_{\text {peak }}$ show an increasing, oscillatory pattern with distinct peaks near $f=0.8,1.6$, and 2.5 (Fig. 8A). This pattern is more pronounced for the $U_{\text {peak }} . U_{\text {peak }}$ greatly exceed the compression wave speed, $c$. $U_{\text {peak }}$ is triple the wave speed near $f=2.5$.

Flow speeds within the compression tube for the racetrack design. $u_{m}$ and $u_{x}$ also share a non-linear relationship with the contraction frequency, $f$. This graph is characterized by oscillations, but peak flow speeds within the compression tube do not correspond to the pattern seen within the racetrack (Fig. 8B).

Flow speeds for the no-racetrack design. $U_{\text {avg }}$ and $U_{\text {peak }}$ show a similar non-linear relationship with $f$ when $c$ is held constant (the racetrack and no-racetrack designs are compared in Fig. 9B). Peaks in both speeds are seen around $f=0.8,1.6$, and 2.5 . Values of $U_{\text {peak }}$ are consistently above $c$.

Volume Flow Rate. For the no-racetrack design, the volume flow rate, $v_{\text {avg }}$, has a non-linear relationship with compression frequency, $f$, when $c$ is held at 3.0 (Fig. 9D).

Pressure. Fig. 7D shows the relationship between pressures, $p_{\text {in }}^{\prime}, p_{\text {out }}^{\prime}$ and $\Delta p$, and frequency, $f$, for constant wave speed (gray items). Pressures show a non-linear relationship with frequency, as in the case of fluid flow speed.

\section{DISCUSSION}

\subsection{Evaluating large-amplitude, short-wave peristaltic pumps against the technical defi- nition of peristalsis}

Männer et al (2010) summarizes 6 qualities of technical peristaltic pumps often used to evaluate biological pumps based on small-amplitude and/or long-wave approximation of peristalsis. Our direct, numerical simulation of peristalsis adheres to a simpler, more inclusive definition of peristalsis: having a nonstationary compression wave that travels uni-directionally down a tube with no structurally fixed direction 
A

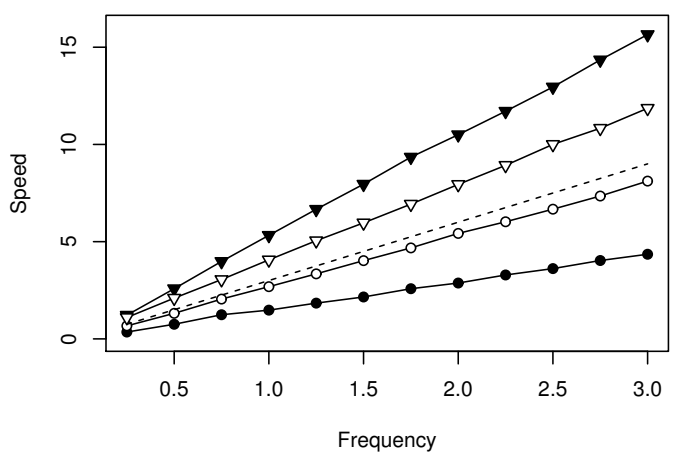

C

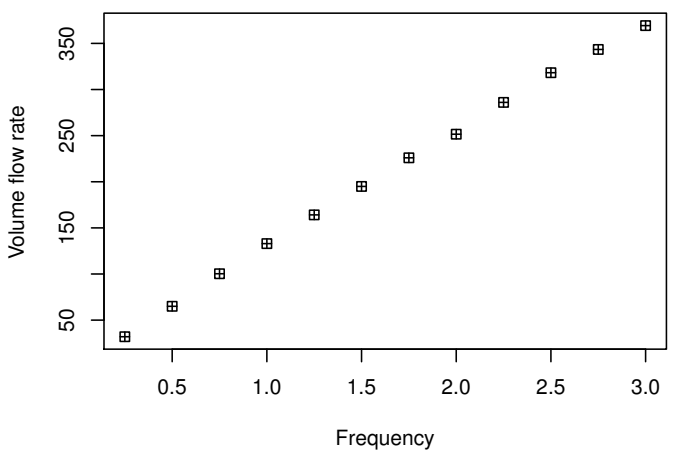

B

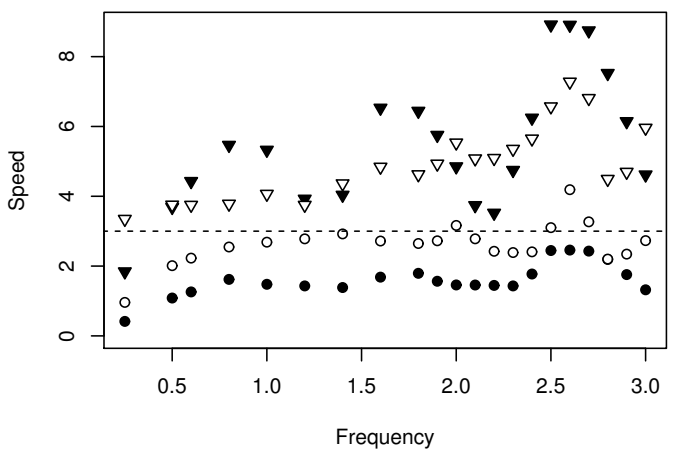

D

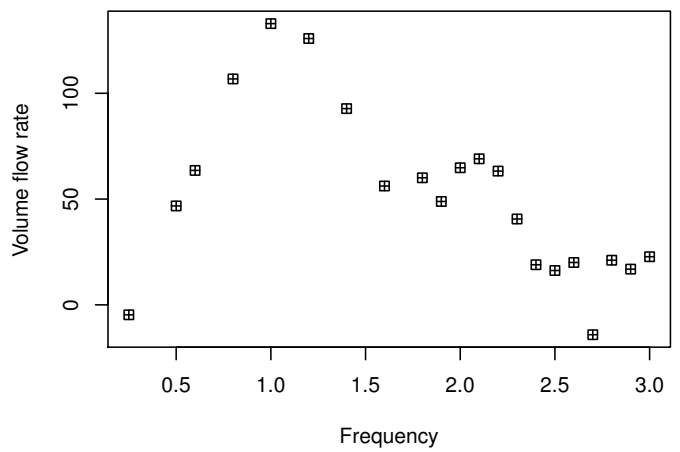

Figure 9. Top row: Non-dimensional fluid speed vs. non-dimensional compression wave frequency, $f$, for two cases: with a connected racetrack (black items) and with no circulatory system or rigid ends (white items). Positive speed indicates movement of fluid in the counter-clockwise direction around the racetrack. $U_{\text {avg }}$ (circles) and $U_{\text {peak }}$ (inverted triangles) measured in the upper tube of the racetrack (racetrack) and the mouth of the contracting region (no racetrack). Dotted lines report the speed of the compression wave, $c$. A. Simulations with variable $c$. B. Simulations with constant $c$. Bottom row: Volume flow rate, $v_{\text {avg }}$, versus compression-wave frequency, $f$, for C. simulations with with variable $c$, and D. simulations with constant $c$.

of flow. The geometry considered in this paper violates the long-wave approximation (the full wavelength is 4 times the diameter for the base case) and the small-amplitude approximation (compressions up to 95\% are considered). Furthermore, the $W o$ was varied from 0.1 to 50 , spanning cases where both inertia and viscosity are significant. Note that this model was not developed to capture the specific dynamics of any particular tubular heart but rather to consider highly nonlinear dynamics.

Our models incorporates the most basic features of peristalsis and produces flow characteristics that run counter to those listed in the technical definition. Our model generates pulsatile fluid flow for Wo of 5 and less at high compression ratios (Fig. 3A and B); flow speeds that show non-linear relationships when either the compression wave speed or the pulsing frequency is fixed (Fig. 5C); and peak flow speeds that exceed compression wave speeds in most cases (Figs. 3A-C, 5A-C, and 9A-B). These results are similar to the results of other valveless, peristaltic models with cardiac cushions (Taber et al, 2007). When flow speeds are measured in the compression section, significant back flow can be observed.

Additionally, the model shows that flow speeds can have either a linear or non-linear relationship with compression frequency, depending on how the speed of the compression wave is handled. A linear relationship between flow speed and compression frequency results when the speed of the compression wave increases linearly with compression frequency (Figs. 8A and 9A,C), demonstrating that flow is being driven by peristalsis and not another pumping mechanism. In this case, the wave form along the 
tube is the same, and the pump just operates faster. This is a feature of most mechanical pumps that does not necessarily translate to biological pumps. In the case of a constant wave speed, the relationship is strikingly non-linear between fluid speed and compression frequency (Fig. 8A and 9B,D). For these cases, the wave form along the compression region changes (demonstrated in Fig. 2). This scenario corresponds to situations when the pacemaker activity is altered while the propagation speed of an action potential is constant.

\subsection{Implications of large-amplitude, short-wave peristaltic pumps in biological fluid trans- port systems}

Since many of the principles of the technical definition derived from mechanical pumps are violated by our simple model, we suggest that the technical definition of peristalsis is inappropriate for evaluating the pumping mechanism of biological pumps. While the definition appropriately describes some peristaltic pumps, many biological pumps may fail simply because they violate the low-amplitude, long-wave approximation used to establish the criteria in the technical definition. Simply decoupling compression wave speed and frequency can also cause a pump to violate the technical definition even though it retains the essential features of peristalsis (traveling wave of compression and no structurally fixed flow direction) that mathematicians and biologists use.

There are many biological pumps that fail criteria in the technical definition of peristalsis but fit the more inclusive definition. In tunicate hearts, heart beat frequency increases with increasing ambient temperature but the conduction velocity that passes down the heart tube remains constant (Kriebal, 1967). In mosquito hearts, hemolymph flow is not continuous and flow speed is greater than compression wave speed (Glenn et al, 2010). For many tubular pumps, the contraction wave may nearly or even completely occlude the tube. Other tubular pumps, such as the embryonic hearts of tunicates, are not much longer than they are wide. Tubes with diameters on the order of their length violate the long-wave assumption.

\subsection{Significance of the pumping mechanism to the embryonic heart}

In the past 8 years, both peristalsis and dynamic suction pumping have been proposed as the mechanism by which the early embryonic heart tube drives the flow of blood. The important point of this difference for the case of understanding the evolution and development of the heart is the distinction between which regions of the heart actively contract. If tubular hearts pump using dynamic suction pumping, then only one region of the heart actively contracts, and the waves observed are passive and elastic. On the other hand, if the pumping mechanism is peristalsis, then the heart actively contracts along its entire length. This distinction has consequences for how the cardiac conduction system and the musculature develops.

In Forouhar et al (2006), work on embryonic zebrafish hearts, they present observations that demonstrate this valveless, tubular heart violates the technical definition of peristalsis. As we've shown with this simple model of flow being driven by peristalsis, fluid flow can mimic each observation made on the embryonic heart, including pulsatile flow both within the compressing tube (Fig. 4A-C) and far away from the compression tube (Fig. 3A-C); peak flow speeds that exceed compression wave speeds (Fig. 5A-F and 9A-B); and a non-linear relationship between fluid flow and compression frequency that is very similar to the relationship observed in their study (Fig. 8A and 9B). The results of our study suggest that peristalsis cannot be ruled out as the pumping mechanism of the vertebrate embryonic heart, and that the exact pumping mechanism requires further study since both mechanisms can result in similar features for cases with nearly complete occlusion and diameter to length ratios on the order of 1:4.

The only observation that supports rejection of peristalsis as the pumping mechanism for the zebrafish heart is that no active contraction down the length of tube was observed (Forouhar et al, 2006). However, this observation has been disputed by others (Männer et al, 2010; Maes et al, 2011; Goenezen et al, 2012) and was not supported by direct measurement of muscle activation or morphological features that would suggest electrical activation of cardiac muscle was limited to one area of the heart. Many studies show that the architecture required to propagate such a signal are present early during heart development and that conduction throughout the myocardium is itself a critical component of normal cardiogenesis (Paff, 1938; Paff et al, 1964; Rottbauer et al, 2001; Chi et al, 2010).

\section{ACKNOWLEDGEMENTS}

The authors would like to thank Boyce Griffith for his assistance with the use of IBAMR and to William Kier for his thoughtful advice on this project over the years. This work was funded by a NSF DMS 
CAREER \# 1151478 (to L. Miller) and by a NSF DMS Research and Training Grant \# 5-54990-2311 (to R. McLaughlin, R. Camassa, L. Miller, G. Forest, and P. Mucha).

\section{REFERENCES}

Anderson M (1968) Electrophysiological studies on initiation and reversal of the heart beat in Ciona intestinalis. J Exp Biol 49:363-385

Aranda V, Cortez R, , Fauci L (2011) Stokesian peristaltic pumping in a three-dimensional tube with a phase-shifted asymmetry. Physics of Fluids 23:081,901

Auerbach D, Moehring W, Moser M (2004) An analytic approach to the liebau problem of valveless pumping. Cardiovascular Engineering: An International Journal 4(2):201-207

Avrahami I, Gharib M (2008) Computational studies of resonance wave pumping in compliant tubes. Journal of Fluid Mechanics 608:139-160

Baird A, King T, Miller LA (2014) Numerical study of scaling effects in peristalsis and dynamic suction pumping. Contemporary Mathematics 628:129-148

Berger MJ, Colella P (1989) Local adaptive mesh refinement for shock hydrodynamics. J Comput Phys 82(1):64-84

Berger MJ, Oliger J (1984) Adaptive mesh refinement for hyperbolic partial-differential equations. J Comput Phys 53(3):484-512

Bringley T, Childress S, Vandenberghe N, Zhang J (2008) An experimental investigation and a simple model of a valveless pump. Physics of Fluids 20:033,602

Ceniceros HD, Fisher JE (2012) Peristaltic pumping of a viscoelastic fluid at high occlusion ratios and large weissenberg numbers. Journal of Non-Newtonian Fluid Mechanics 171-172(31-41)

Chi N, Bussen M, Brand-Arzamendi K, Ding C, Olgin J, Shaw R, Martin G, Stainier D (2010) Cardiac conduction is required to preserve cardiac chamber morphology. Proc Natl Acad Sci U S A 107(33): 14,662-14,667

Childress S (2009) An Introduction to Theoretical Fluid Mechanics, Courant Lecture Notes, vol 19. American Mathematical Society

Childs H, Brugger E, Whitlock B, Meredith J, Ahern S, Pugmire D, Biagas K, Miller M, Harrison C, Weber GH, Krishnan H, Fogal T, Sanderson A, Garth C, Bethel EW, Camp D, Rübel O, Durant M, Favre JM, Navrátil P (2012) VisIt: An End-User Tool For Visualizing and Analyzing Very Large Data. In: High Performance Visualization-Enabling Extreme-Scale Scientific Insight, pp 357-372

Christoffels VM, Moorman AFM (2009) Basic science for the clinical electrophysiologist. Circulation: Arryhythmia and Electrophysiology 2:195-207

Davidson B (2007) Ciona intestinalis as a model for cardiac development. Semin Cell Dev Biol 18(1):1626

Forouhar AS, Liebling M, Hickerson A, Nasiraei-Moghaddam A, Tsai HJ, Hove JR, Fraser SE, Dickinson ME, Gharib M (2006) The embryonic vertebrate heart tube is a dynamic suction pump. Science 312(5774):751-753

Fung YC, Yih CS (1968) Peristaltic transport. ASME E J Appl Mech 35:669-675

Gashev A (2002) Physiologic aspects of lymphatic contractile function. Annals of the New York Academy of Sciences 979:178-187

Glenn J, King J, Hillyer J (2010) Structural mechanics of the mosquito heart and its function in bidirectional hemolymph transport. J Exp Biol 213:541-550

Goenezen S, Rennie M, Rugonyi S (2012) Biomechanics of early cardiac development. Biomech Model Mechanobiol 11:1187-1204

Greenlee K, Socha J, Eubanks H, Thapa G, Pederson P, Lee W, Kirkton S (2013) Hypoxia-induced compression in the tracheal system of the tobacco hornworm caterpillar, Manduca sexta L. J Exp Biol 216:2293-2301

Griffith B (2014) An adaptive and distributed-memory parallel implementation of the immersed boundary (ib) method. URL https://github.com/IBAMR/IBAMR

Griffiths D, Constantinou C, Mortensen J, Djurhuus J (1987) Dynamics of the upper urinary tract: II. the effect of variations of peristaltic frequency and bladder pressure on pyeloureteral pressure/flow relations. Phys Med Biol 32(7):832-833

Hanin M (1968) The flow through a channel due to transversely oscillating walls. Israel J Tech 6:67-71 
Harrison J, Waters J, Cease A, Cease A, VandenBrooks J, Callier V, Klok C, Shaffer K, Socha J (2013a) How locusts breathe. Physiology 28:18-27

Harrison JF, Waters JS, Cease AJ, VandenBrooks JM, Callier V, Klok CJ, Shaffer K, Socha JJ (2013b) How locusts breathe. Physiology 28(1):18-27

Hickerson AI, Rinderknecht D, Gharib M (2005a) Experimental study of the behavior of a valveless impedance pump. Experiments in fluids 38(4):534-540

Hickerson AI, Rinderknecht D, Gharib M (2005b) Experimental study of the behavior of a valveless impedance pump. Experiments in Fluids 38(4):534-540

Jaffrin M, Shapiro A (1971) Peristaltic pumping. Annual Review of Fluid Mechanics 3:13-37

Jung E, Peskin CS (2000) Two-dimensional simulations of valveless pumping using the immersed boundary method two-dimensional simulations of valveless pumping using the immersed boundary method. SIAM J Sci Comput 23(1):19-45

Jung E, Lee S, Lee W (2008) Computational models of valveless pumping using the immersed boundary method. Computer Methods in Applied Mechanics and Engineering 197:2329-2339

Kalk M (1970) The organization of a tunicate heart. Tissue and Cell 2:99-118

Krenn H (2010) Feeding mechanisms of adult Lepidoptera: structure, function, and evolution of the mouthparts. Annu Rev Entomol 55:307-327

Kriebal M (1967) Conduction velocity and intracellular action potentials of the tunicate heart. J General Physiology 50(8):2097-2107

Lee W, Socha J (2009) Direct visualization of hemolymph flow in the heart of a grasshopper (Schistocerca americana. BMC Physiology 9(2):doi:10.1186/1472-6793-9-2

Lee W, Lim S, Jung E (2012) Dynamical motion driven by periodic forcing on an open elastic tube in fluid. Commun Comput Phys 12:494-514

Liebau G (1954) Über ein ventilloses pumpprinzip. Naturwissenschaften 41:327-327, DOI 10.1007/BF00644490, URL http://dx.doi.org/10.1007/BF00644490

Liebau G (1955) Die stromungsprinzipien des herzens. Z Kreislaufforsch 44:677

Liebau G (1957) Die bedeutung der tragheitskrafte fur die dynamik des blutkreislaufs. Z Kreislaufforsch $46: 428$

Maes F, Chaudhry B, Ransbeeck PV, Verdonck P (2011) The pumping mechanism of embryonic hearts. IFMBE Proceedings 37:470-473

Männer J, Wessel A, Yelbuz T (2010) How does the tubular embryonic heart work? looking for the physical mechanism generating unidirectional blood flow in the valveless embryonic heart tube. Developmental Dynamics 239:1035-1046

Paff G (1938) The beahvior of the embryonic heart in solutions of ouabain. Am J Physiol 122(3):753-758

Paff G, Boucek R, Klopfenstein H (1964) Experimental heart-block in the chick embryo. Anatomical Record 149:217-223

Peskin CS (2002) The immersed boundary method. Acta Numer 11:479-517

Postma AV, Christoffels VM, Moorman AFM (2008) Developmental aspects of the electrophysiology of the heart: Function follows form. Electrical Diseases of the Heart pp 24-36

Pozrikidis C (1987) A study of peristaltic flow. J Fluid Mech 180:515-527

Rottbauer W, Baker K, Wo Z, Mohideen M, Cantiello H, Fishman M (2001) Growth and function of the embryonic heart depend upon the cardiac-specific L-type calcium channel $\alpha 1$ subunit. Developmental Cell 1:265-275

Santhanakrishnan A, Miller LA (2011) Fluid dynamics of heart development. Cell Biochem Biophys 61(1):1-22

Shapiro A, Jaffrin M, Weinberg S (1969) Peristaltic pumpuing with long wave lengths at low reynolds number. J Fluid Mech 37:799-825

Taber LA (2001) Biomechanics of cardiovascular development. Annu Rev Biomed 3:1-25

Taber LA, Zhang J, Perucchio R (2007) Computational model for the transition from peristaltic to pulsatile flow in the embryonic heart tube. J Biomech Eng 129:441-449

Team RDC (2011) R: A Language and Environment for Statistical Computing. R Foundation for Statistical Computing, Vienna, Austria, http://www.r-project.org/ edn

Teran J, Fauci L, Shelley M (2008) Peristaltic pumping and irreversibility of a stokesian viscoelastic fluid. Physics of Fluids 20:073,101

Vogel S (2007) J Biosci 32(2):207-222 
Waldrop L, Miller LA (2015) The role of the pericardium in the valveless, tubular heart of the tunicate, ciona savignyi, submitted.

Xavier-Neto J, Castro R, Sampaio A, Azambuja A, Castillo H, Cravo R, Simoes-Costa M (2007) Parallel avenues in the evolution of hearts and pumping organs. Cell Mol Life Sci 64:719-734

Xavier-Neto J, Davidson B, Simoes-Costa M, Castillo H, Sampaio A, Azambuja A (2010) Heart Development and Regeneration, vol 1, 1st edn, Elsevier Science and Technology, London, chap Evolutionary origins of the heart, pp 3-38 